\title{
Parabolized Stability Analysis of Jets Issuing from Serrated Nozzles
}

\author{
Aniruddha Sinha ${ }^{1}$, Hao Xia ${ }^{2} \&$ Tim Colonius $^{3}$ \\ ${ }^{1}$ Aerospace Engineering, Indian Institute of Technology Bombay, Mumbai, 400076, INDIA \\ ${ }^{2}$ Aeronautical Engineering, Loughborough University, Leicestershire LE11 3TU, UK \\ ${ }^{3}$ Engineering and Applied Sciences, California Institute of Technology, Pasadena, CA, USA \\ E-mail: as@aero.iitb.ac.in
}

\section{INTRODUCTION}

The mixing noise in jet engine exhaust is one of the main noise sources in aviation. Nozzle lip serrations, commonly called chevrons, are one of the technologies deployed to address the issue in modern civilian aircraft. These nozzle serrations impinge on the shear layer and change its dynamics; the jet plume becomes lobed or serrated near the nozzle in a time-averaged sense, but reverts to a round profile further downstream. A parametric study of subsonic (Mach 0.9) turbulent jets issuing from serrated nozzles was conducted at the NASA SHJAR facility [1]. It demonstrated that such a device reduces the low frequency mixing noise aft of the jet (the peak noise component), while increasing the high frequency omnidirectional noise. The latter component is commonly attributed to enhancement of small-scale turbulence by the impingement of the chevron tips on the shear layer. The low-frequency louder component is less well understood, but is usually linked to the large-scale coherent structures present within the turbulent shear layer. Here we propose a model for these coherent structures in a serrated jet, based on the theory of parabolized stability equations (PSE).

Large-scale coherent structures in shear layers are commonly modeled as instability waves of the timeaveraged turbulent flow field [2]. This idea had been explored first by Crighton \& Gaster [3], and by numerous researchers since. However, the theory has been recently placed on a surer footing by repeated validation against the detailed experimental/computational databases that are currently becoming available $[4,5,6]$. The first attempt at linear stability theory (LST) usually makes a parallel-flow assumption, and this was indeed pursued by Gudmundsson \& Colonius [7] for the serrated jet. PSE is a less-restrictive theory, as it allows for a gradual streamwise variation in the base flow.

\section{PSE MODEL}

In the PSE model of the serrated jet, the fluctuation $q^{\prime}$ of any flow variable $q$ around its time average $\bar{q}$ is expressed

$$
q^{\prime}(x, r, \theta, t)=\hat{q}_{m, \omega}(x, r) \exp \left\{i\left(\int_{x_{0}}^{x} \alpha_{m, \omega}(\xi) d \xi+m \theta-\omega t\right)\right\},
$$

where $\hat{q}$ is a shape function (with 'minimal variation in $x$ ), and $\alpha$ is the complex axial wavenumber with mild axial variation. Stationarity and azimuthal periodicity allow the corresponding Fourier transforms indicated. This ansatz is substituted into the governing equations for a compressible flow linearized about $\bar{q}$, and the PSE assumption of mild $x$-variation is invoked so that the solution can be marched in $x$, instead of needing to be solved globally (in $x \& r$ ). The PSE solution march is initiated with a parallel-flow LST solution. The details of the implementation appear elsewhere [6].

The resulting model for a base flow with general variation is a set of equations that is dense in $m$, the azimuthal Fourier mode; Uzun et al. [8] have recently adopted this approach for the serrated jet. However, the nozzle serrations are always rotationally symmetric, so that the serrated base flow also possesses this simplicity. Ref. [7] used this observation to partially decouple the serrated jet LST problem in the azimuthal Fourier domain, and we adopt the same idea for the PSE model herein. In particular, with 6 chevrons (as in the SMC001 nozzle tested at NASA [1], and also modeled here), there are four independent solutions to the PSE problem; (a) $m \in\{\ldots,-12,-6,0,6,12, \ldots\}$, (b) $m \in\{\ldots,-11,-5,1,7,13, \ldots\},(\mathrm{c})$ $m \in\{\ldots,-10,-4,2,8,14, \ldots\}$, and (d) $m \in\{\ldots,-9,-3,3,9, \ldots\}$.

\section{ILLUSTRATIVE RESULTS}

The near irrotational hydrodynamic field of a turbulent jet has the best footprint of the instability waves [2]. Hence, it is best to look for validation of the PSE model thereat. Xia et al. [9] developed an LES database of the Mach 0.9 cold jet issuing from the SMC001 nozzle tested at NASA. We process this database to extract the pressure fluctuations on a virtual conical array of pressure sensors, at the modeled frequencies and azimuthal modes (placed as in the NASA SHJAR experiments [10]). A proper orthogonal decomposition (POD) of the pressure data [4] is performed to abstract the coherent part of the signal for validation of the PSE model. Figure 1 shows the result of this exercise at some of the acoustically important frequencies (expressed in Strouhal number $S t$ ) and azimuthal modes. 

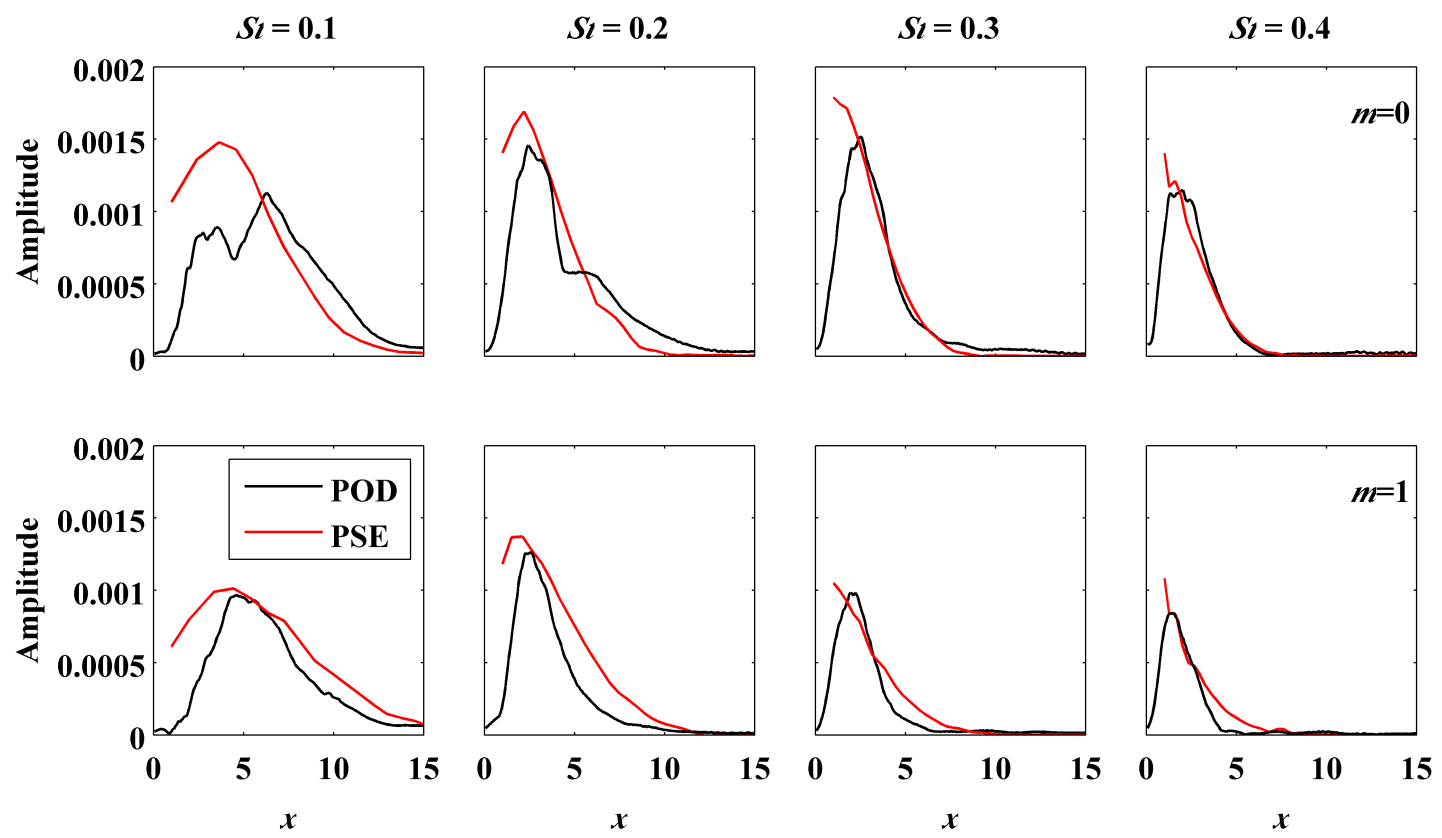

Figure 1: Amplitude of pressure along virtual microphone array placed in near hydrodynamic field of jet (as in the NASA SHJAR experiments [1]). The POD-filtered data is compared with the scaled model predictions.

The model predictions are encouraging for $0.2 \leq S t \leq 0.4$, for both the axisymmetric $(m=0)$ and first helical $(m=1)$ modes. The instability wave model has been repeatedly found to be inadequate for lower frequencies. The near-nozzle portion of the predicted pressure signature is not predicted accurately. This may be due to the transitional nature of this region of the jet in the LES.

The final paper will report more details of the method and results.

\section{REFERENCES}

[1] J. E. Bridges and C. A. Brown, "Parametric testing of chevrons on single flow hot jets," in 10th AIAA/CEAS Aeroacoustics Conference, AIAA Paper 2004-2824, 2004.

[2] P. Jordan and T. Colonius, "Wave packets and turbulent jet noise," Annu. Rev. Fluid Mech., vol. 45, pp. 173-195, 2013.

[3] D. G. Crighton and M. Gaster, "Stability of slowly diverging jet flow," Journal of Fluid Mechanics, vol. 77, no. 2, pp. 397-413, 1976.

[4] K. Gudmundsson and T. Colonius, "Instability wave models for the near-field fluctuations of turbulent jets," Journal of Fluid Mechanics, vol. 689, pp. 97-128, 2011.

[5] A. V. G. Cavalieri, D. Rodríguez, P. Jordan, T. Colonius, and Y. Gervais, "Wavepackets in the velocity field of turbulent jets," Journal of Fluid Mechanics, vol. 730, pp. 559-592, 2013.

[6] A. Sinha, D. Rodríguez, G. Brès, and T. Colonius, "Wavepacket models for supersonic jet noise," Journal of Fluid Mechanics, vol. 742, pp. 71-95, 2014.

[7] K. Gudmundsson and T. Colonius, "Spatial stability analysis of chevron jet profiles," in 13th AIAA/CEAS Aeroacoustics Conference, AIAA Paper 2007-3599, 2007.

[8] A. Uzun, F. S. Alvi, T. Colonius, and M. Y. Hussaini, "Spatial stability analysis of subsonic jets modified for low-frequency noise reduction." Submitted.

[9] H. Xia, P. G. Tucker, and S. J. Eastwood, "Large-eddy simulations of chevron jet flows with noise predictions," Int. J. Heat and Fluid Flow, vol. 30, pp. 1067-1079, 2009.

[10] T. Suzuki and T. Colonius, "Instability waves in a subsonic round jet detected using a near-field phased microphone array," Journal of Fluid Mechanics, vol. 565, pp. 197-226, 2006.

\section{KEYWORDS}

Instability wavepackets, parabolized stability equations, coherent structures, jets, chevroned jets. 